\title{
CARACTERIZAÇÃO DA DEMANDA DE FONOAUDIOLOGIA NO SERVIÇO PÚBLICO MUNICIPAL DE RIBEIRÃO DAS NEVES - MG
}

\section{Characterizing the demand of speech-language pathology in the Municipal Public Service of Ribeirão das Neves - MG}

\author{
Andréa de Melo César ${ }^{(1)}$, Simone Siqueira Maksud ${ }^{(2)}$
}

\begin{abstract}
RESUMO
Objetivo: caracterizar a população local encaminhada ao Serviço de Fonoaudiologia do Núcleo de Atenção Psicopedagógico Infanto-juvenil - NAPPI, além de oferecer uma contribuição para futuras pesquisas em Fonoaudiologia no âmbito da saúde pública. Métodos: o estudo foi realizado a partir da coleta e análise descritiva de dados referentes ao período de acolhimento, origem do encaminhamento, faixa etária, gênero e queixas fonoaudiológicas das crianças da fila de espera. Resultados: dos 161 encaminhamentos, a maioria foi acolhida no período de agosto a dezembro de $2004 ; 41 \%$ foram realizados por médicos, sendo o gênero masculino representado por $66 \%$ das crianças, com idade entre cinco e dez anos (60\%). De acordo com as queixas apresentadas, as mais freqüentes foram as alterações de fala (46\%), as de linguagem (18\%) seguidas pela motricidade oral $(15 \%)$. Com relação ao número de queixas por encaminhamento, uma queixa representa $38 \%$, duas queixas $53 \%$ e $9 \%$, três queixas associadas. Conclusão: o perfil da demanda fonoaudiológica no Município de Ribeirão das Neves écaracterizado pela prevalência do gênero masculino com idade entre cinco e dez anos, encaminhados em sua maioria por médicos, apresentando freqüentemente duas queixas associadas, sendo a principal as alterações de fala.
\end{abstract}

DESCRITORES: Saúde Pública; Fonoaudiologia; Promoção da Saúde; Prevenção de Doenças

\section{INTRODUÇÃO}

A Fonoaudiologia, como uma ciência recente, carece de estudos que possam produzir conhecimentos técnicos e científicos capazes de expandir seu domínio de atuação em saúde pública ${ }^{1}$. Entre os anos setenta e oitenta, os fonoaudiólogos foram inseridos no sistema público pelas Secretarias de Educação e Saúde. Os serviços geralmente eram estruturados como um consultório, marcado por práticas reabilitadoras ${ }^{2,3}$. Pesquisas no campo da Fonoaudiologia comunitária tornam-se relevantes, a fim de oferecer contribuição para definir melhor o papel e lugar do fonoaudiólogo junto à promoção da saúde da população de maneira reflexiva, consciente, responsável e atuante ${ }^{2,4}$. O profissional de saúde pública deve ter por objetivo a pro-

(1) Fonoaudióloga do Núcleo de Atenção Psicopedagógico Infanto-Juvenil - NAPPI; Especialista em Motricidade Oral.

(2) Fonoaudióloga do Núcleo de Atenção Psicopedagógico Infanto-Juvenil - NAPPI; Especialista em Motricidade Oral. moção, prevenção e recuperação da saúde fonoaudiológica da população, tendo interesse nas doenças que afetam um grupo específico, mas aplicando medidas de caráter comunitário ${ }^{2,5}$. Para isto, é preciso conhecer as condições de saúde deste grupo, por meio da caracterização da população usuária dos serviços coletivos por meio de estudos epidemiológicos, a fim de elaborar políticas adequadas à população ${ }^{6,7}$.

A ação do fonoaudiólogo no serviço público requer capacidade de percepção das alterações fonoaudiológicas que acometem a população em geral, como também das peculiaridades e dinamicidade da demanda local onde o profissional está lotado.

Inicialmente, o fonoaudiólogo tentou adotar a estrutura de atendimento clínico-privado no sistema público, sendo os resultados não satisfatórios. Desta maneira, começou a buscar novos caminhos que levassem a um melhor desempenho nos vários níveis de atenção à saúde ${ }^{8}$.

A atuação fonoaudiológica no contexto da saúde pública implica uma mudança marcada por práticas 
assistencialistas e reabilitadoras para a capacidade de elaborar e efetivar ações que visem sua solução, bem como adotar medidas preventivas cabíveis, prestando um atendimento de qualidade à população ${ }^{9-11}$.

Este estudo tem por objetivo caracterizar a população local encaminhada ao serviço de Fonoaudiologia do Núcleo de Atenção Psicopedagógico Infanto-juvenil NAPPI, já que a carência de informações epidemiológicas na área dificulta o planejamento e organização de ações fonoaudiológicas mais eficazes. Pretende-se, também, oferecer uma contribuição para futuras pesquisas em Fonoaudiologia comunitária.

\section{MÉTODOS}

O NAPPI, até o período do estudo, é o único núcleo da cidade de Ribeirão das Neves a oferecer serviços de fonoaudiologia para os usuários da rede pública. Este núcleo tem como critério para acolhimento, crianças até 17 anos de idade residentes no município.

Foram analisadas todas as fichas de pacientes da fila de espera acolhidos pelo setor de fonoaudiologia do NAPPI, totalizando 161 acolhimentos datando o período de janeiro de 2003 a junho de 2005.

A coleta e análise dos dados foram realizadas por duas fonoaudiólogas, num mesmo momento, por meio de protocolo específico. As informações levantadas foram referentes ao período de acolhimento, compreendido pelo primeiro e segundo semestre dos anos de 2003 e 2004 e primeiro semestre do ano de 2005; origem dos encaminhamentos: escolar médico e outros; faixas etárias, divididas em: 0 a 5 anos, 5 a 10 anos, 10 a 15 anos e maiores de 15 anos de idade; gênero; queixas fonoaudiológicas apresentadas, classificadas em alterações de fala, linguagem, motricidade orofacial, distúrbios de aprendizagem, deficiência auditiva, disfemia e disfonia e o número de queixas por encaminhamento: uma, duas ou três queixas associadas. Consideramos os distúrbios de fala às alterações que se restringem ao domínio fonêmico, correspondendo a realização motora da lin- guagem. Compreende as alterações que afetam a produção dos sons da língua. Estes distúrbios estão ligados principalmente às fases de programação e execução neuromotora. Os distúrbios de linguagem dizem respeito às alterações que afetam todo o desenvolvimento lingüístico da criança, aos fatores pragmáticos, formal ou semântico, estando ligados ao conteúdo ${ }^{12-15}$. Já os distúrbios de motricidade orofacial, são as alterações anatômicas e/ou funcionais do sistema sensório motor oral, comprometendo as funções realizadas pelo sistema estomatognático. ${ }^{16,17}$

O presente estudo foi avaliado pelo Comitê de Ética em Pesquisa do Centro de Especialização em Fonoaudiologia Clínica, sob número 216/06.

Os resultados foram analisados por meio de estatística descritiva com freqüência absoluta e relativa, sendo representados por gráficos.

\section{RESULTADOS}

Foram totalizados 161 encaminhamentos na fila de espera, sendo 55 (35\%) acolhidos entre agosto e dezembro de 2004, 47 (29\%) entre janeiro e julho deste mesmo ano, 31 (19\%) referente ao período de janeiro a junho de 2005, seguido por 22 (14\%) entre agosto a dezembro de 2003 e 5 (3\%) correspondendo aos meses de janeiro a julho de 2003. Quanto à origem dos encaminhamentos, $41 \%$ são realizados por Médicos e $40 \%$ por escolas, seguidos por profissionais da Terapia Ocupacional, Enfermagem, Psicologia e Odontologia totalizando 19\%. Desses, nove encaminhamentos não têm especificações sobre a origem (Figura 2). O gênero masculino representa $66 \%$ dos pacientes enquanto 0 feminino 34\% (Figura 3). Quanto à faixa etária, 60\% têm idade entre 05 a 10 anos, seguido por 0 a 05 anos (20\%), 10 a 15 anos (15\%) e maiores de 15 anos (5\%) (Figura 4). De acordo com as queixas apresentadas, as mais freqüentes são as alterações de fala (46\%), as de linguagem (18\%) e de motricidade oral (15\%) (Figura 5). Com relação ao número de queixas por encaminhamento, uma queixa representa $38 \%$, duas queixas $53 \%$ e $9 \%$, três queixas associadas (Figura 6).

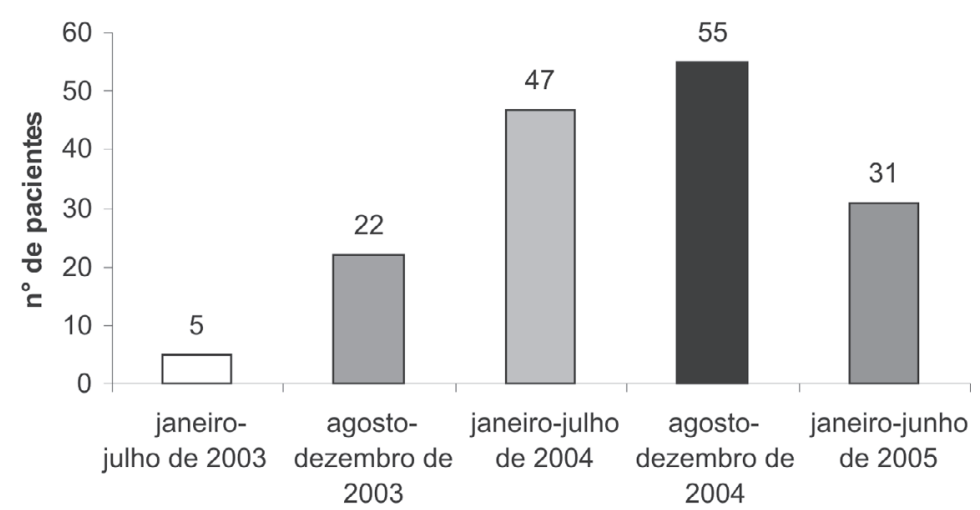

Figura 1 - Distribuição dos pacientes da fila de espera de acordo com o período de acolhimento 


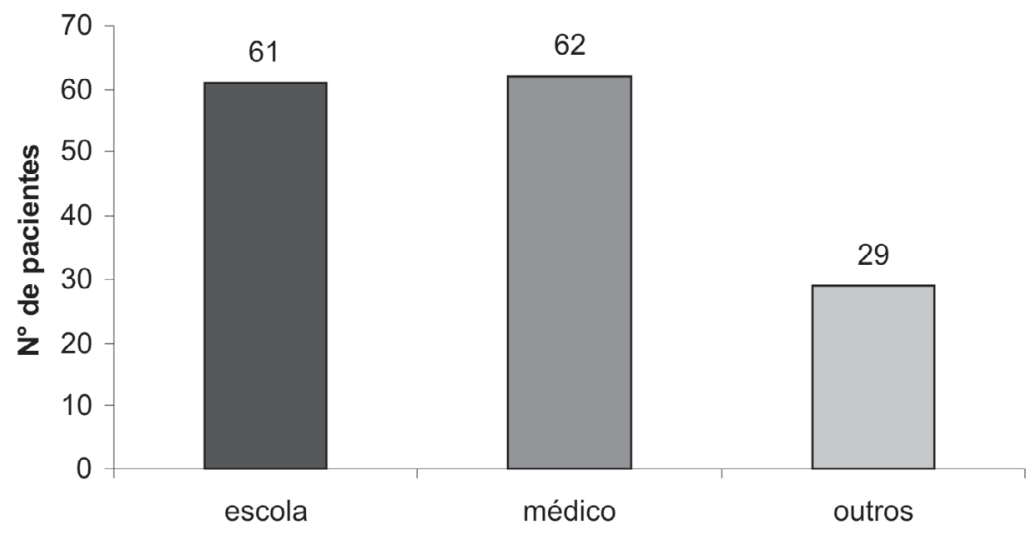

Figura 2 - Origem do encaminhamento de pacientes da fila de espera

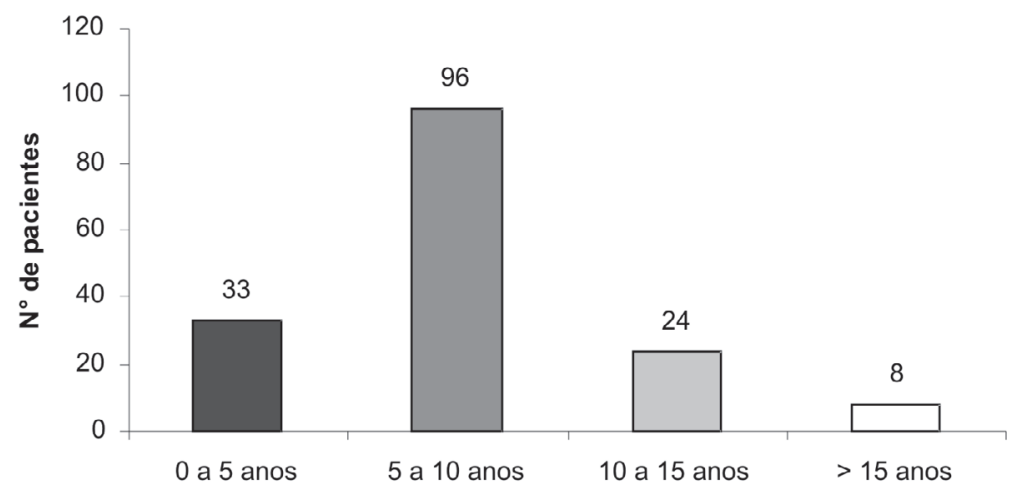

Figura 3 - Distribuição dos pacientes da fila de espera de acordo com a faixa etária

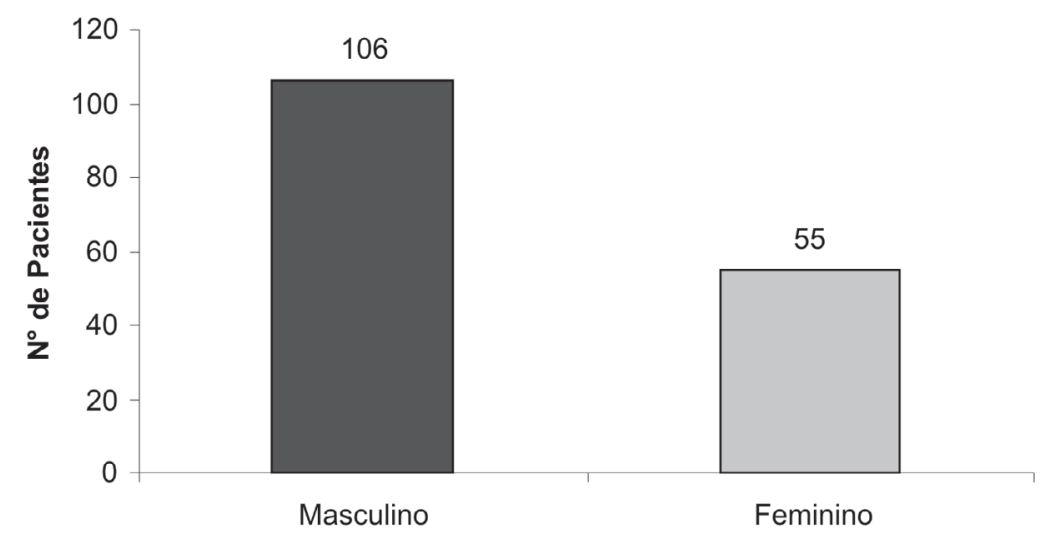

Figura 4 - Distribuição dos pacientes da fila de espera de acordo com o gênero 


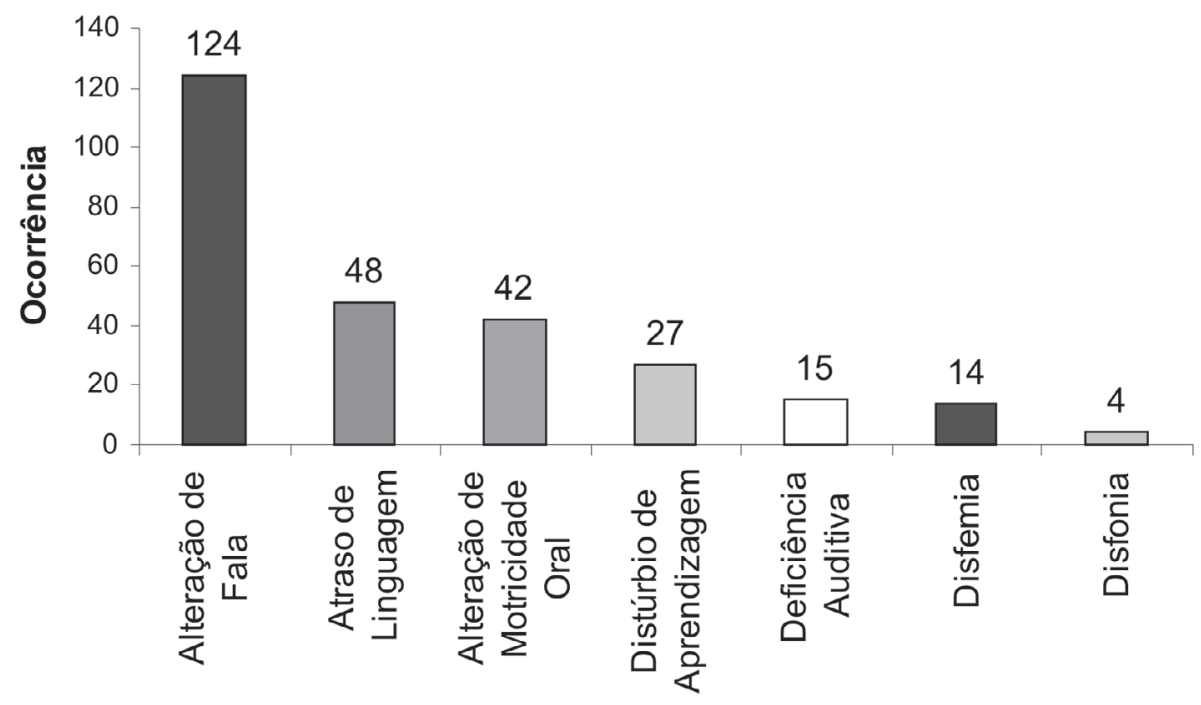

Figura 5 - Ocorrência de alterações fonoaudiológicas independente do número de queixas apresentadas

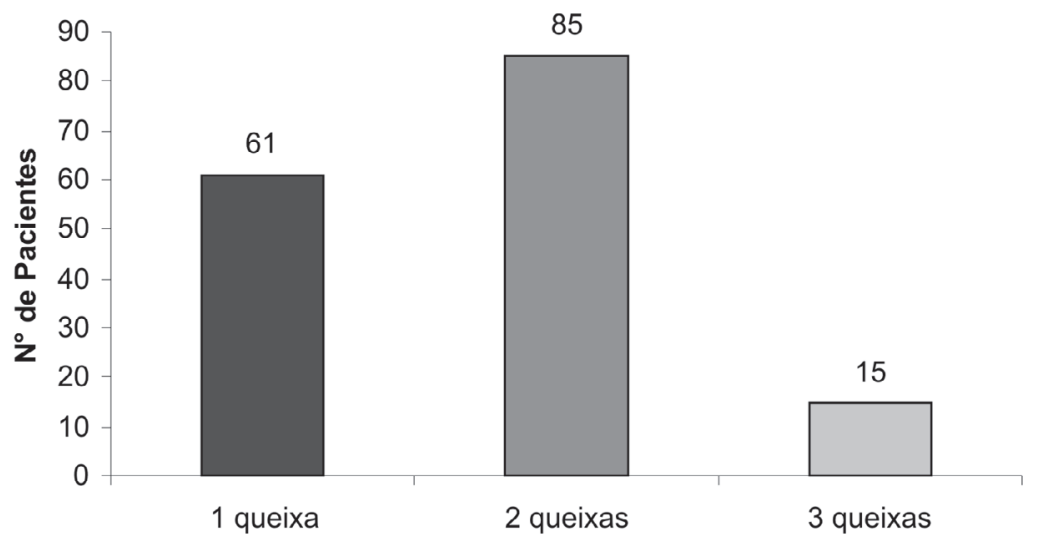

Figura 6 - Número de queixas fonoaudiológicas apresentadas por encaminhamento

\section{DISCUSSÃO}

Durante o período de estudo, observou-se que no ano de 2004 foi realizado maior número de acolhimentos no setor de fonoaudiologia. Este dado pode ser justificado pelo fato dos pacientes da fila de espera, acolhidos em 2003, já estarem em fonoterapia ou já terem sido atendidos, resultando em um número menor que o ano de 2004.

Quanto à origem dos encaminhamentos, quase houve uma igualdade entre os pacientes encaminhados por médicos e pelas escolas. Alguns estudos encontraram maior ocorrência por parte das escolas, seguida pelos postos de saúde ${ }^{6,9}$. Outro estudo relata prevalência dos encaminhamentos médicos ${ }^{18}$. O índice de encaminhamentos médicos justifica-se talvez pelo julgamento dos pais de ser este o meio pos- sível para registro formal de suas queixas e solicitação de avaliação especializada. Encaminhamentos escolares podem tender a um número mais elevado à medida que a divulgação e conhecimento do serviço prestado pelo NAPPI forem se ampliando pelas escolas do município, por meio de projetos já em andamento. A capacitação de professores sobre o desenvolvimento normal de linguagem, fala e audição bem como o fornecimento de orientações sobre aspectos de domínio da Fonoaudiologia, certamente terá influência futura no número de encaminhamentos.

O gênero masculino predominou sobre o feminino, achados também observados em estudos anteriores $^{6,9,18-21}$. Investigações mais detalhadas deveriam ser realizadas, já que esta prevalência de alterações no gênero masculino é referida em vários levantamentos fonoaudiológicos, sem que haja 
explicações comprovadas. A interpretação convencional para este fato é que o cérebro dos meninos apresenta uma maturação mais lenta que o das meninas ${ }^{18,22}$. Fatores genéticos também têm sido apontados como determinantes na prevalência de distúrbios específicos de linguagem no gênero masculino ${ }^{23,24}$. Outros estudos também mostraram prevalência da população entre cinco a dez anos 6,18 , seguido por zero a cinco anos ${ }^{2,6,9}$. A demanda maior nesta faixa etária, fase pré-escolar e escolar, pode ser justificada pelo ingresso na escola, local onde as alterações de fala e linguagem são frequentemente percebidas, já que a criança inicia novas interações sociais e são mais cobradas quanto ao seu desempenho da linguagem oral e aprendizagem ${ }^{6}$. As alterações de fala (46\%) tiveram maior ocorrência, seguidas pelas alterações de linguagem (18\%) e de motricidade oral (15\%), fato também encontrado em outro estudo ${ }^{3}$. Outras pesquisas encontraram como alterações mais freqüentes as de fala, ${ }^{6,9,19}$ seguida das alterações de linguagem e de aprendizagem ${ }^{6}$. Outra pesquisa encontra seguida das alterações de fala, as alterações de motricidade orofacial, os distúrbios de aprendizagem e alterações de linguagem ${ }^{6}$. Acredita-se que as ocorrências de motricidade oral poderiam ser maiores, já que a população do município não tem contato estreitado com o profissional ortodontista, grande fonte de encaminhamentos nesta especialidade fonoaudiológica.
A presença de mais de uma queixa fonoaudiológica foi comum entre os acolhimentos, sendo que a maioria apresentou duas queixas associadas. Não encontramos outros estudos que analisaram o número de queixas fonoaudiológicas apresentadas.

A análise destes registros permitiu aos fonoaudiólogos adotarem ações mais eficazes e coerentes com as necessidades locais, como a implementação de grupos terapêuticos e de orientações aos pais direcionados especificamente ao perfil da demanda. Além disso, houve aprimoramento da dinâmica de atendimento e maior interlocução interinstitucional por meio de consultoria às escolas municipais, objetivando estreitar relações com professores e educadores infantis.

\section{CONCLUSÃO}

Por meio deste estudo foi possível traçar o perfil da demanda por atendimento fonoaudiológico no município de Ribeirão das Neves - MG, sendo o mesmo caracterizado pela prevalência do gênero masculino com idade entre cinco a dez anos, encaminhado em sua maioria por médicos, apresentando freqüentemente duas queixas associadas, sendo a principal as alterações de fala.

A caracterização dessa demanda, comparada à de outros serviços possibilitará traçar com maior critério a atuação do fonoaudiólogo junto às instituições e a criação de políticas mais abrangentes no contexto da Saúde Pública.

\begin{abstract}
Purpose: to characterize the local population directed to the service of Speech Pathology of Núcleo Psicopedagógico Infanto-Juvenil- NAPPI, in addition to offering a contribution for future research in Speech Language Pathology in the scope of the public health. Methods: the study was carried on children' screening waiting line of NAPPI. A descriptive analysis of the referring data, regarding the period of screening, origin of the guiding, age, sex and speech pathology complaints, was carried out. Results: within 161 guides, the majority $(41 \%)$ were done by doctors, where the male gender represented $66 \%$ of the children, with age between five and ten years $(60 \%)$. In accordance with the submitted complaints, the most frequent were alterations of speech (46\%), followed by alterations of language $(18 \%)$ and orofacial myology (15\%). With relation to the number of complaints per guide, one complaint represents $38 \%$, two complaints $53 \%$ and $9 \%$ three associated complaints. Conclusion: the profile of the Speech-Language Pathology demand in the city of Ribeirão das Neves is characterized by the prevalence of male gender, with age between the five and ten years, directed in its majority by doctors and presenting two associated complaints. The most frequent one was speech alterations (46\%).
\end{abstract}

KEYWORDS: Public Health; Speech, Language and Hearing Sciences; Health Promotion; Disease Prevention

\section{REFERÊNCIAS}

1. Mendes VLF. Fonoaudiologia e saúde coletiva: perspectivas de atuação nos serviços públicos de saúde. Distúrb Comun. 1999; 10(2):213-24.
2. Pereira MTJG. Fonoaudiologia: uma vivência em saúde pública [monografia]. São Paulo (SP): Centro de Especialização em Fonoaudiologia Clínica; 1999. 3. Marin CR, Chun RYS, Silva RC, Fedosse E, Leonelli BS. Promoção da saúde em fonoaudiologia: 
ações coletivas em equipamentos de saúde e de educação. Rev Soc Bras Fonoaudiol. 2003; 8(1):35-41. 4. Penteado RZ, Servilha EAM. Fonoaudiologia em saúde pública / coletiva: compreendendo prevenção e o paradigma da promoção da saúde. Distúrb Comun. 2004; 16(1):107-16.

5. Dahan D. Atuação fonoaudiológica em saúde pública: uma abordagem preventiva. [periódico on-line] Disponível em:

URL: http://www.fonoaudiologia.com/trabalhos/artigos/artigo-026.htm Acesso em: 21 out 2006.

6. Gonçalves CGO, Lacerda CBF, Perotino S, Mugnaine AMM. Demanda pelos serviços de fonoaudiologia no município de Piracicaba: estudo comparativo entra a clínica escola e o atendimento na prefeitura municipal. Pró-fono. 2000; 12:61-6.

7. Gonçalves MS, Tochetto TM, Primo MT. Fonoaudiologia e saúde coletiva: prioridades detectadas pelos usuários de unidades básicas de saúde. Rev Fonoaudiol Bras. 2005; 3(2):1-3.

8. Wertzner HF. Ambulatórios de fonoaudiologia em Unidade Básica de Saúde. In: Befi D, organizador. Fonoaudiologia na atenção primária à saúde. Atualidades em Fonoaudiologia vol III. São Paulo: Lovise; 1997. p.161-76.

9. Freire RM. Fonoaudiologia em saúde pública. Rev Saúde Pública. 1992; 26:179-84.

10. Souza RPF, Cunha DA, Silva HJ. Fonoaudiologia: a inserção da área de linguagem no Sistema Único de Saúde (SUS). Rev CEFAC. 2005; 4:426-32.

11. Befi D. A inserção da fonoaudiologia na atenção primária à saúde. In: Befi $D$, organizador. Fonoaudiologia na atenção primária à saúde. Atualidades em Fonoaudiologia vol III. São Paulo: Lovise; 1997. p. 15-35.

12. Zorzi JL. Aspectos básicos para compreensão, diagnóstico e prevenção dos distúrbios de linguagem na infância. Rev CEFAC. 2000; 2:111-5.

13. Schirmer CR, Fontoura DR, Nunes ML. Distúrbios da aquisição da linguagem e da aprendizagem. J Pediatr. 2004; 2:95-102.

14. Zorzi JL. Os problemas de fala. [periódico online] Entrevista concedida para revista Âmbito Farmacêutico. Disponível em:
URL: $\quad$ http://www.cefac.br/library/artigos/ a40e249ff5ec147c74448d366914a301.pdf

15. Zorzi JL. Distinguiendo alteraciones del habla y del lenguaje. [periódico on-line] CEFAC. Disponível em : URL:www.cefac.br/library/artigos/ 38d6434cc634a8b3f448c6ebfe966320.pdf

16. Marchesan IQ. Distúrbios da motricidade oral. In: Russo ICP. Intervenção fonoaudiológica na terceira idade. Rio de Janeiro: Revinter; 1998. p.83-100.

17. Marchesan IQ. Avaliando e tratando o sistema estomatognático. In: Campiotto AR, Levy C, Holzheim D, Rabinovich K, Vicente LCC, Castiglioni M, Redondo MC, Anelli W. Tratado de fonoaudiologia. São Paulo: Roca; 1997. p. 763-80.

18. Hage SRV, Faiad LNV. Perfil de pacientes com alteração de linguagem atendidos na clínica de diagnóstico dos distúrbios da comunicação - Universidade de São Paulo - Campus Bauru. Rev CEFAC. 2005; 7(4):433-9.

19. Andrade CRF. Prevalência das desordens idiopáticas da fala de da linguagem em crianças de um a onze anos de idade. Rev Saúde Pública. 1997; 31:495-501.

20. Rapin I, Allen DA, Dunn MA. Developmental language disorders. In: Segalowitz SJ, Rapin I, organizadores. Handbook of neurophychology. v. 7. New York: Elsevier; 1992. p. 111-37

21. Choudhury N, Benasich AA. A family aggregation study: the influence of family history and other risk factors on language development. J Speech Lang Hea Res. 2003; 46(2):261-72.

22. Geschwind N, Galaburda AM. Cerebral lateralization. Biological mechanisms, associations, and pathology: III. A hyphotesis and a program of research. Arch Neurol. 1985; 42(3):428-59.

23. Lai CS, Fisher SE, Hurst JA, Vargha-Khadem F, Monaco AP. A forkhead-domain gene is mutatedin a severe speech and language disorder. Nature. 2001; 413(6855):519-23.

24. O'Brien EK, Zhang X, Nishimura C, Tomblin JB,Murray JC. Association of specific language impairment (SLI) to the region of $7 q 31$. Am J Hum Genet. 2003; 72(6):1536-43.

\section{RECEBIDO EM: 03/10/06 \\ ACEITO EM: 04/02/07}

Endereço para correspondência:

Rua Nelson Soares Faria, 83 / 201

Belo Horizonte - MG

CEP: 31170030

E-mail: andreamcesar@yahoo.com.br 
